\title{
PHOTOCHEMICAL TRANSFORMATIONS OF DIVINYL ARENES
}

\author{
J. Meinwald, J. W. Young, E. J. Walsh and A. Courtin \\ Department of Chemistry, Cornell University, Ithaca, \\ New York 14850, U.S.A.
}

\begin{abstract}
Continuing earlier studies on the photochemistry of 1,3,5-hexatriene and $o$-divinylbenzene, the photochemical isomerization of four divinylnaphthalene derivatives has been examined. Irradiation of 1,2-divinylnaphthalene leads to a bicyclo[3.1.0]-hexene derivative, while 2,3-divinylnaphthalene reacts analogously, but, as anticipated, also gives a small amount of a naphthobicyclo[2.1.1]hexene, providing the first example of a head-to-tail cyclization of the terminal vinyl groups in the series. 1,8-Divinylnaphthalene is isomerized smoothly to a $10: 1$ ratio of head-to-tail and head-to-head cyclization products, perinaphthobicyclo[3.1.1] heptene and peri-naphthobicyclo[3.2.0] heptene. The previously reported selective photocyclization of 1,8-distyrylnaphthalene to a high-melting peri-naphthobicyclo[3.2.0]heptene is shown to be in error; this product is actually a symmetrical dimer. Irradiation of dilute solutions of 1,8-distyrylnaphthalene, however, does give three monomeric isomers; the major two products possess nead-to-tail peri-naphthobicyclo[3.1.1] heptene skeletons, while the minor product results from a head-to-head cyclization. In these reactions, the major products correspond to expectations based on the most favourable conformations of the unexcited starting material. These reactions hold promise for the synthesis of a variety of strained ring systems.
\end{abstract}

The chemistry of highly strained ring systems has intrigued us for many years ${ }^{1,2}$, and since photochemical techniques are often especially appropriate for the synthesis of strained molecules, we have become involved in some photochemical studies as well. As an example, we have explored a number of routes to the synthesis of the simplest bridged olefin, bicyclo[2.1.1] hexene (I). While this objective was ultimately realized ${ }^{3}$, some of our unsuccessful exploratory experiments were interesting in their own right, and led to the series of investigations which form the main body of this report.

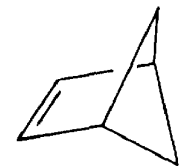

(])

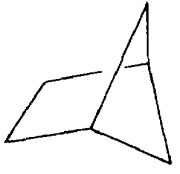

(II)
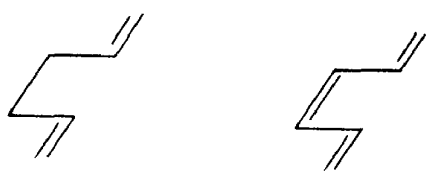

(III) 
It was Srinivasan who showed that bicyclo[2.1.1]hexane (II) can be obtained from the mercury-sensitized photocyclization of 1,5 -hexadiene (III) ${ }^{4}$. This suggested that $1,3,5$-hexatriene (IV) might cyclize similarly to give (I), although Srinivasan's vapour phase work with (IV) was not encouraging ${ }^{5}$. Nevertheless, a subsequent report that upon irradiation in solution $\alpha$-phellandrene (V) is transformed, via the corresponding acyclic triene ${ }^{6}$, into a bicyclo[2.1.1] hexene derivative (VI) ${ }^{7}$, stimulated us to examine this possibility further.

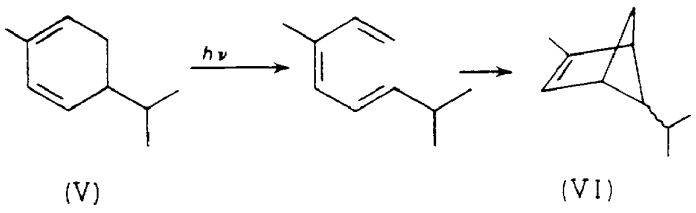

Re-examination of the photochemistry of (V) revealed that the chief monomeric products formed by cyclization of the acyclic triene were the isomeric exo- and endo-bicyclo[3.1.0]hexenes (VII) and (VIII) ${ }^{8}$. While this

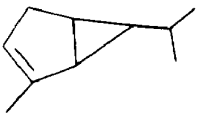

(VII)

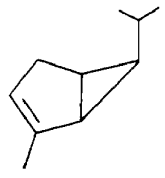

(VIII)

mode of cyclization finds precedent in a wide variety of relatively complex molecules, we were surprised to learn that the solution photochemistry of 1,3,5-hexatriene itself (or of the corresponding 1,3-cyclohexadiene) had not been looked at from this point of view. Irradiation of 1,3-cyclohexadiene in fact gave a mixture of bicyclo[3.1.0]hexene (IX) and 3-vinylcyclobutene

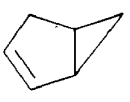

(IX)

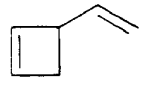

$(\mathrm{X})$

(X) rather than the desired (I) ${ }^{9}$. Thus, the outlook for a criss-cross (head-totail) cyclization between the terminal portions of a conjugated triene system did not appear good.

On the basis of this experience, we wondered whether the replacement of the central double bond of a conjugated triene system by an aromatic ring might change this pattern of reaction, giving rise to cyclization products in

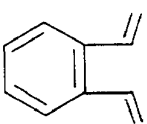

(XI)

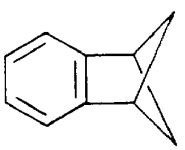

(XII)<smiles>c1ccc2c(c1)C1CCC21</smiles>

(XIII) 


\section{PHOTOCHEMICAL TRANSFORMATIONS OF DIVINYL ARENES}

which the aromatic nucleus would remain undisturbed. Were this to happen, photochemical rearrangement of $o$-divinylbenzene (XI) might be expected to yield benzobicyclo[2.1.1] hexene (XII) or benzobicyclo[2.2.0]hexene (XIII). In fact, irradiation of $(\mathrm{XI})$ does bring about an isomerization, but contrary to expectation, the product proved to be benzobicyclo[3.1.0]hex-2-ene (XIV), a result most easily interpreted as involving the exact analogy of the $(\mathrm{IV}) \rightarrow(\mathrm{IX})$ conversion, followed by a vinylcyclopropane $\rightarrow$ cyclopentene rearrangement which re-aromatizes the non-benzenoid intermediate (A) thus produced ${ }^{10,11}$.

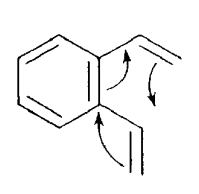

$(X I)$

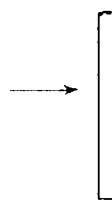

$(\mathrm{A})$<smiles>c1ccc2c(c1)CC1CCC2C1</smiles>

$(X I V)$

If the formation of $(A)$ is actually involved in the photochemical rearrangement of (XI) to (XIV), there are some interesting consequences that might be expected in the photochemistry of the naphthalene analogues of (XI). Thus, 1,2-divinylnaphthalene (XV), with greater double bond character between the carbon atoms bearing the vinyl groups than exists in the case of (XI), would surely be expected to cyclize smoothly either via intermediate (B) to give 1,2-naphthobicyclo[3.1.0]hex-2-ene $(\mathrm{XV})$, or else via $\left(\mathrm{B}^{\prime}\right)$ to give $(\mathrm{XVII})$. It is important to note here that both $(\mathrm{B})$ and $\left(\mathrm{B}^{\prime}\right)$ retain one aromatic ring intact.

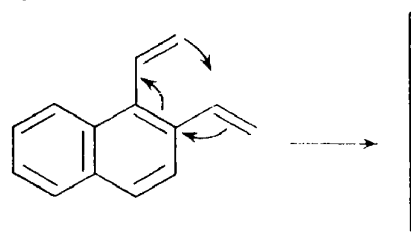

$(\mathrm{XV})$

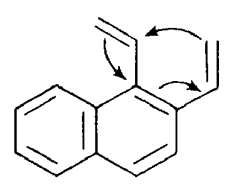

$(X V)$

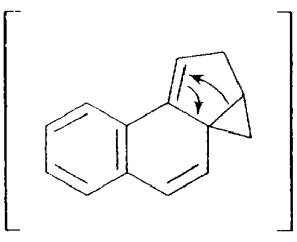

(B)
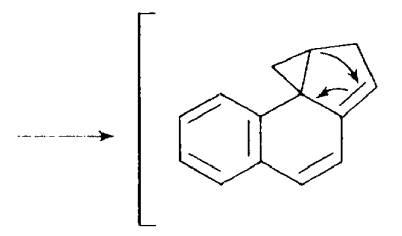

(B')

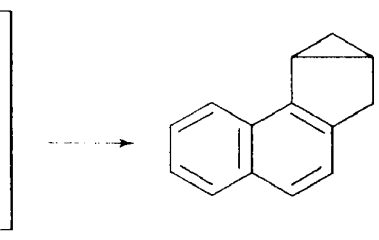

(XV1)

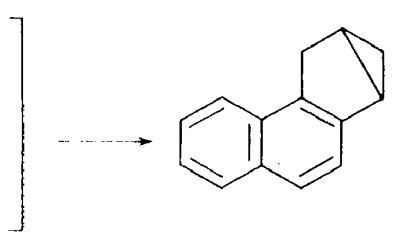

(XVII)

In contrast, consideration of possible photoisomerizations of 2,3divinylnaphthalene (XVIII) reveals that if this compound were to follow the same reaction pattern, it would have to generate an intermediate $(C)$ in which the aromaticity of both rings is lost on the way to the 2,3-naphthobicyclo[3.1.0]hex-2-ene (XIX). Under these circumstances, the alternative 


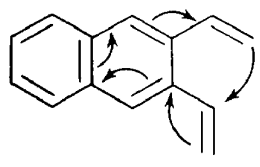

(XVIII)
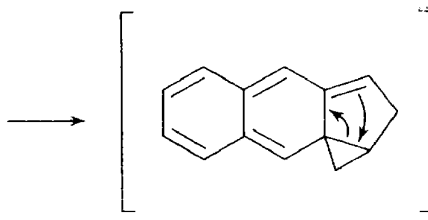

(C)

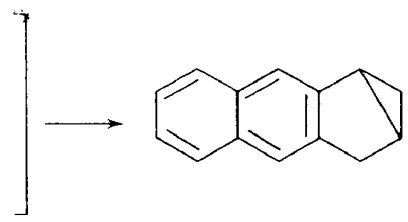

$(X I X)$

possibilities of cycloadditions leading to 2,3-naphthobicyclo[2.1.1] hex-2-ene (XX) or 2,3-naphthobicyclo[2.2.0]hex-2-ene (XXI), either of which would require no loss of aromaticity, might be expected to become observable, if not predominant, reactions.<smiles>c1ccc2cc3c(cc2c1)C1CCC3C1</smiles>

$(X X)$<smiles>c1ccc2cc3c(cc2c1)C1CCC31</smiles>

$(X X I)$

Finally, we became intrigued with the extension of these photoisomerization studies to the case of 1,8-divinylnaphthalene (XXII), the only remaining divinylnaphthalene sterically capable of cycloaddition between the vinyl groups. Since the vinyl substituents in (XXII) are separated by three carbon atoms rather than two, no pathway analogous to the formation of (IX) from (IV) [or (A), (B), (B') and (C) from (XI), (XV) and (XVIII)] exists. Consequently, if (XXII) were to suffer photochemical rearrangement, peri-naphthobicyclo[3.1.1] heptene (XXIII) and perinaphthobicyclo[3.2.0]heptene (XXIV) would appear to be the most likely products. In pursuing these studies, we were interested not only in ascertaining the course of the photochemical reactions, but also in the possibility of exploiting them for synthetic purposes, since the chemistry of ring systems such as those present in (XXIII) and (XXIV) has been essentially unexplored.

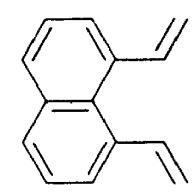

(XXII)

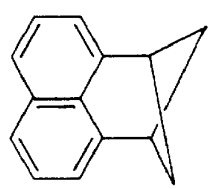

(XXIII)

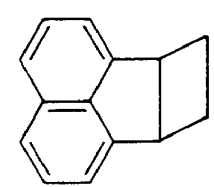

(XXIV)

The synthesis of 1,2-divinylnaphthalene (XV) is outlined in Chart I. While the procedure is somewhat lengthy, there are no special difficulties. The structure of (XV) was confirmed by the usual spectral methods. Irradiation of a dilute solution of (XV) in pentane gave some insoluble, polymeric material, along with a comparable amount of monomeric products. GLC resolved this mixture into three components, present in a $5: 90: 5$ ratio. The minor component, of longest retention time, was readily 
PHOTOCHEMICAL TRANSFORMATIONS OF DIVINYL ARENES Chart I<smiles>C=C(Br)c1ccccc1</smiles><smiles>C=Cc1ccc2cc(C)ccc2c1C=C</smiles><smiles>O=C(CCCl)c1ccc2ccccc2c1</smiles><smiles>OC1CCc2c1ccc1ccccc21</smiles><smiles>C1=Cc2ccc3ccccc3c2C1</smiles><smiles>Cc1ccc2c3c(ccc2c1)C1CC(C3)C1</smiles>

(XVII)

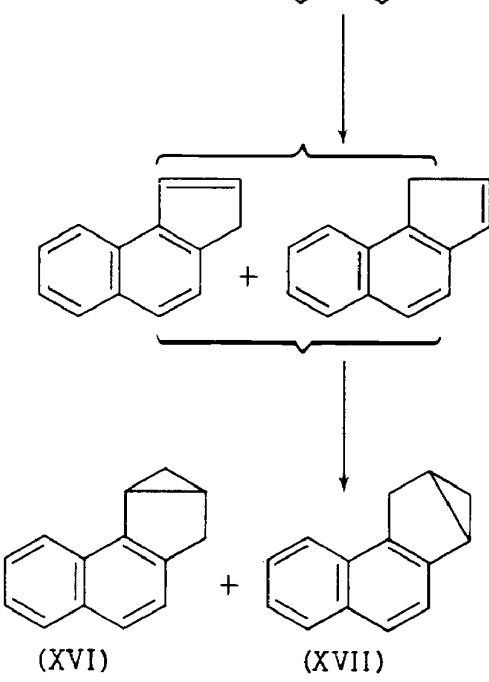


identified as phenanthrene, apparently resulting from a head-to-head bonding mechanism followed by oxidative aromatization. The other minor product could not be obtained sufficiently pure to permit characterization. The major product was recognized as one of the two possible 1,2-naphthobicyclo[3.1.0]hexenes (XVI) or (XVII) on the basis of spectral evidence, especially its characteristic n.m.r. spectrum. The decision between structures (XVI) and (XVII) was difficult, however, and could not be made on any simple spectroscopic basis. An unambiguous synthesis of (XVII) was therefore devised, as shown in Chart II. The product of this synthesis was similar to, but (from its n.m.r. spectrum) clearly different from the photoproduct in question. In fact, the photoproduct could not have contained more than five per cent of this isomer. While (XVI) was not obtained pure by synthesis, a mixture of (XVI) and (XVII) was readily prepared (see Chart II), and the properties of the major photoproduct were in good accord with those deduced for (XVI) from examination of the unresolved mixture.

We see, then, that the photochemistry of $(\mathrm{XV})$ corresponds well to expectation. The preference for cyclization to give (XVI) rather than (XVII) can be rationalized by consideration of the possible conformations of (XV) which would be responsible for each of these products. Conformation (XVa), which should lead via (B) to (XVI) is no more hindered than the benzenoid analogue (XI), while conformation $(\mathrm{XVb})$, which would have led to $(\mathrm{XVI})$ via $\left(\mathrm{B}^{\prime}\right)$ suffers considerable non-bonded repulsion between a terminal hydrogen of the vinyl group and the $\mathrm{C}_{8}$-peri hydrogen. Of course, the reacting species probably do not have these exactly planar conformations, but these steric considerations do seem to point in the right direction.

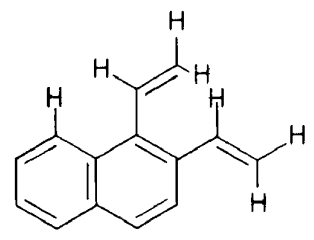

$(\mathrm{XVa})$<smiles>C=Cc1ccc2ccccc2c1</smiles>

$(\mathrm{XVb})$

Chart III outlines the synthesis of 2,3-divinylnaphthalene (XVIII). Irradiation of a one per cent solution of (XVIII) in ether or pentane gave about 50 per cent of insoluble polymer, along with 30 to 50 per cent of monomeric photoproduct. Aside from 2-4 per cent of anthracene, corresponding again to a head-to-head bonding process, an $85: 15$ ratio of photoisomers was obtained. The major isomer, m.pt $67.5-69^{\circ}$, could be assigned structure (XIX) on the basis of unambiguous spectral evidence. The minor product, m.pt $101-102^{\circ}$, was identified as (XX), once again on the basis of the striking similarity of its n.m.r. spectrum to that of benzobicyclo[2.1.1] hexene (XII). Chemical confirmation of this structure was obtained by ozonolysis of (XX), which gave cis-1,3-dicarbomethoxycyclobutane upon oxidative workup followed by diazomethane esterification.

Thus, in spite of the necessity of destroying its entire aromatic system, the major pathway for photochemical rearrangement of (XVIII) involves 


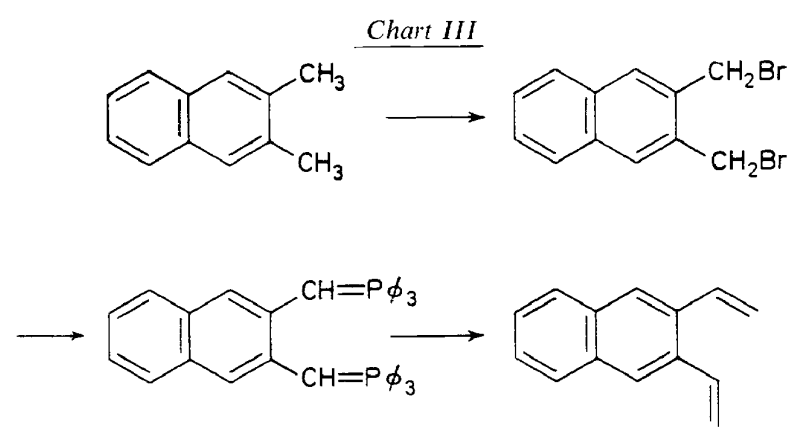

(XVIII)

formation of intermediate (C), in direct analogy to the rearrangements of (XV), (XI) and (IV) itself. However, the long sought alternative of a crisscross cycloaddition leading to the formation of 2,3-naphthobicyclo[2.1.1.] ]hex2-ene (XX), now appears as a competing process for the first time.

In contrast to the divinylnaphthalenes discussed above, 1,8-divinylnaphthalene (XXII) is a known compound ${ }^{12,13}$. Ultra-violet irradiation of dilute solutions of (XXII) resulted in its rapid conversion to a mixture of two difficultly separable isomers in a 10:1 ratio. The major product, m.pt $78^{\circ}$, showed an n.m.r. spectrum strongly reminiscent of the fused bicyclo[2.1.1]hexenes (XII) and (XX), suggesting the fused bicyclo[3.1.1] hexene structure (XXIII). In its mass spectrum, this substance showed, besides a parent ion $\left(\mathrm{M}^{+}\right)$at $m / e$ 180, and $\mathrm{M}-15$ (165) base peak which can be readily rationalized as shown below.
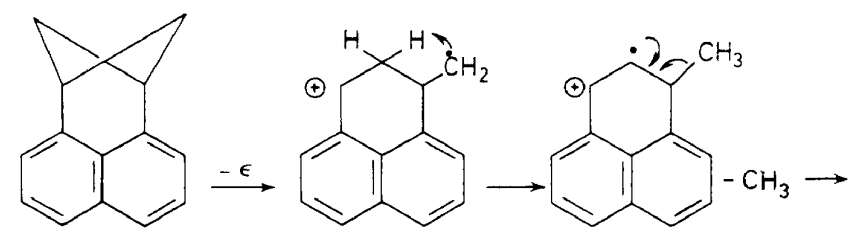

(XXIII)<smiles>[Y15]C1=CC=Cc2cccc3cccc1c23</smiles>

The minor product (m.pt $52^{\circ}$ ) from the irradiation of (XXII) was recognized as the bicyclo[3.2.0] heptane derivative (XXIV) by direct comparison with an authentic sample obtained as outlined in Chart IV ${ }^{14}$. The spectral data for (XXIV) are all in accord with expectation. In particular, the mass spectrum has its base peak at $m / e 152$, resulting from loss of an ethylene fragment (M-28) to give ionized acenaphthalene. These structures were confirmed chemically by ozonolysis of the cyclization products, which gave ciscyclobutane-1,3-dicarboxylic acid and cis-cyclobutane-1,2-dicarboxylic acid 


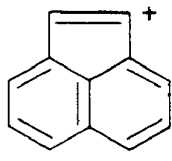

$M-28$

respectively. Thus, in the case of (XXII), the anticipated cyclizations take place, with head-to-tail (criss-cross) orientation predominating.

While the above-described work was in progress, an independent group studying the photochemistry of 1,8-distyrylnaphthalene (XXV) reported a clean-cut transformation to give a head-to-head cycloaddition product (XXVI) of unspecified stereochemistry ${ }^{15}$. It would not be surprising if the<smiles>[O]C=Cc1cccc2cccc(C=C[O-])c12</smiles>

$(\mathrm{XXV})$

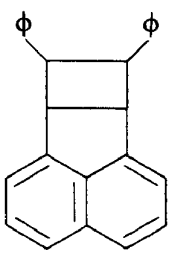

(XXVI)

presence of phenyl substituents in (XXV) were to reverse the preferred direction of cyclization observed for (XXII). Nevertheless, we undertook a further study of this reaction, since the evidence in favour of structure (XXVI) did not appear compelling, and since it appeared worthwhile to ascertain the product's stereochemistry in any event.

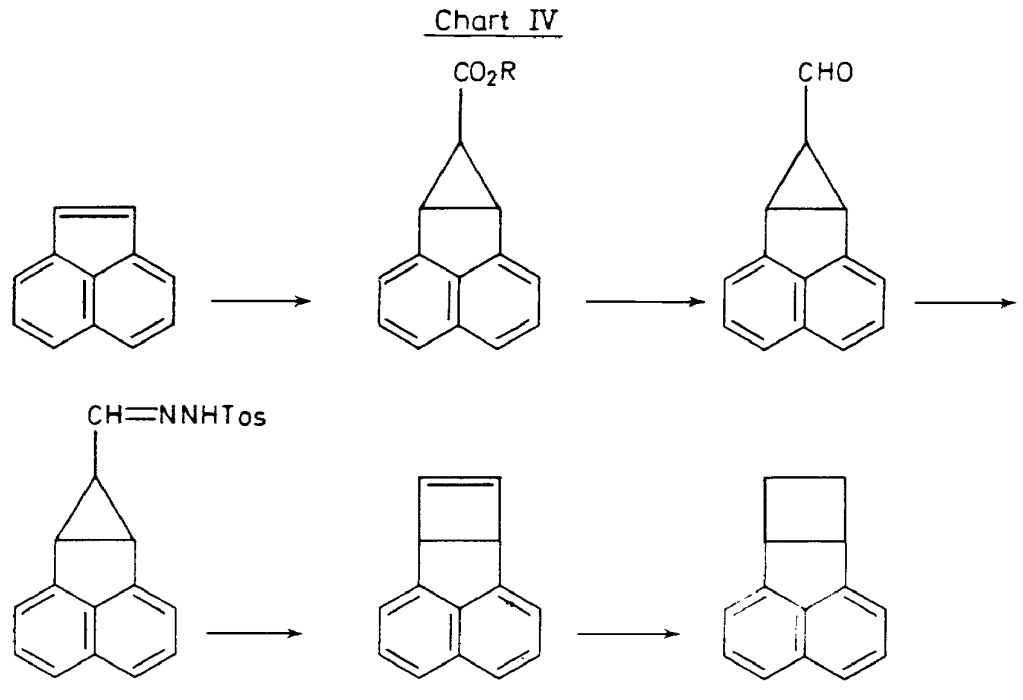


On repeating the irradiation of $(\mathrm{XXV})$ as previously described $(0.15 \mathrm{M}$ solution in refluxing cyclohexane), a high melting (m.pt $335^{\circ}$ ) photoproduct corresponding to the literature report was obtained in good yield. The high melting point and unusually low solubility of this material suggested to us that it might not be a monomeric photoisomer at all. This suspicion was confirmed by both osmometric and mass spectrometric molecular weight determinations, which clearly indicated a dimeric formula.

There are many dimers that might be expected to form photochemically from (XXV). Since it seemed clear on spectral grounds that no double bonds remained in the dimer, all four double bonds were assumed to participate in the dimerization process. Four possible structures are given below: (XXVII), (XXVIII), (XXIX) and (XXX).

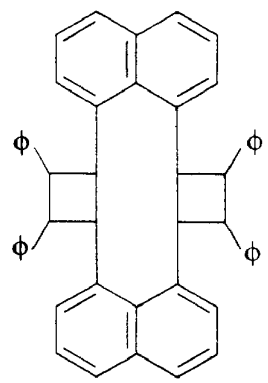

(XXVII)

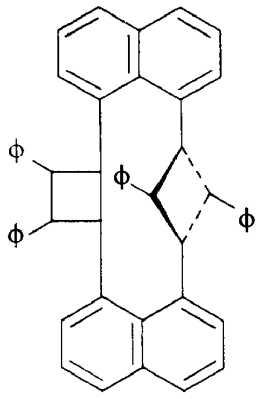

$(\mathrm{XXIX)}$

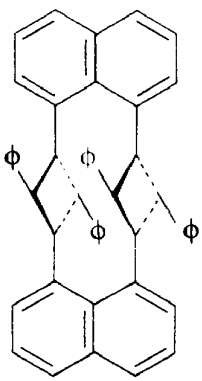

(XXVIII)

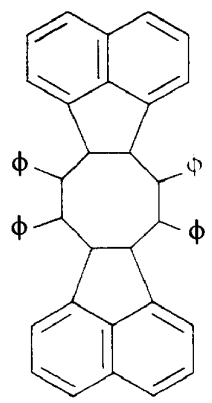

$(\mathrm{XXX})$

The simplicity of the dimer's n.m.r. spectrum excludes the unsymmetrical (XXIX), and the mass spectrum, which, in addition to a parent ion $\left(\mathrm{M}^{+}\right)$at $\mathrm{m} / \mathrm{e} 664$, shows peaks at 484 and 304 , corresponding to the loss of one and two molecules of stilbene, speaks against (XXVIII), (XXIX) and (XXX). Structure (XXVII) therefore appeared most attractive. The chemical experiments described below support this conclusion, and also indicate the dimer's stereochemistry.

Pyrolysis $\left(350^{\circ}\right)$ of the dimer, following a pattern similar to that observed in the mass spectrometer, resulted in the formation of some of the original 
monomer (XXV), along with trans-stilbene and a highly coloured material. The isolation of (XXV), representing simply thermal reversal of the original photochemical dimerization, is compatible with any of the four dimer formulae. The recovery of trans-stilbene, however, combined with the mass spectral evidence already presented, suggested that (XXXI) might be another pyrolysis product, if formula (XXVII) correctly represented the original dimer.

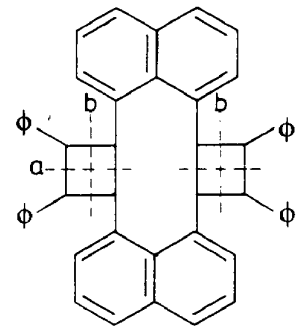

(XXV II)

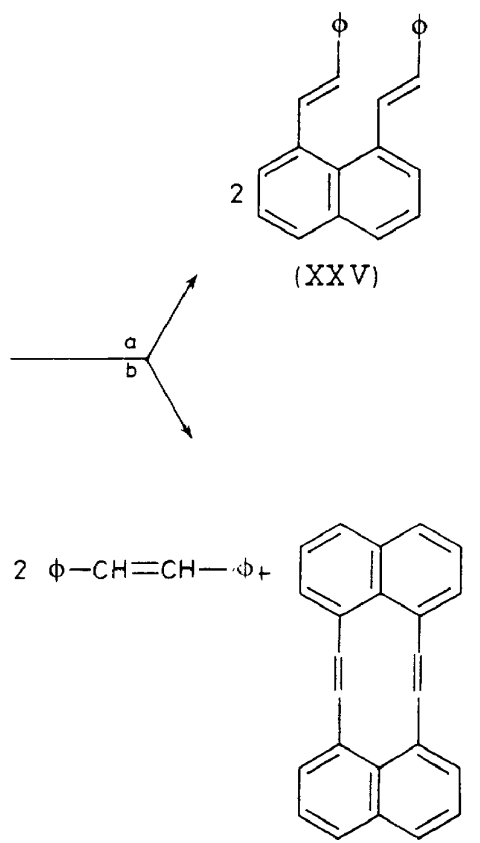

(XXXI)

While (XXXI) itself could not be isolated from the reaction mixture, this olefin is known to be very readily autoxidized ${ }^{16}$ to the deep-red aromatic hydrocarbon zethrene (XXXII) ${ }^{17}$. We were gratified, therefore, to be able to isolate a deep red material from the crude pyrolysate which showed the

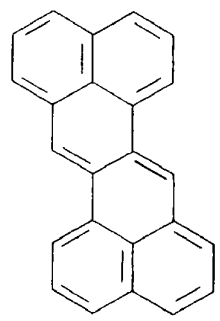


highly characteristic visible absorption spectrum of zethrene, giving strong support for our structure assignment.

An interesting feature of this facile dimerization is the fact that the stereochemistry of (XXVII) corresponds to that of the original 1,8-distyrylnaphthalene units (trans,trans), as established by exhaustive ozonolysis. Thus, the smooth degradation of the dimer to the known cis,trans,cis-1,2,3,4tetracarbomethoxycyclobutane (XXXVIII) permits its formulation as (XXVIIa).

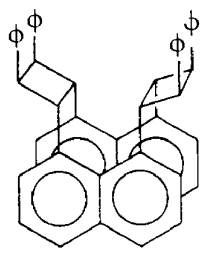

(XXVII a)

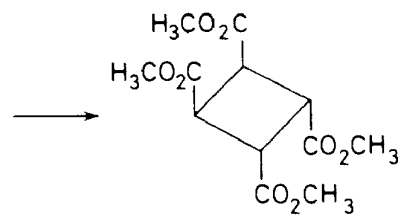

$(\mathrm{XXXIII)}$

Two recent publications call attention to apparently analogous dimerizations of $o$-divinylbenzene derivatives. In the case of the trans,trans-diester (XXXIV), a 0.1 per cent yield of a dimer containing no double bonds has been reported by Tavares and Ploder ${ }^{18}$. Because of the very low yield, the structure of this product has not yet been ascertained. However, Müller, Meier and Sauerbier ${ }^{19}$ were able to show that prolonged irradiation of concentrated solutions of trans, trans-o-distyrylbenzene (XXXV) gave a mixture of saturated dimers, in which the main isomer was assigned formula (XXXVI) on the basis of n.m.r. spectral analysis. It would appear from these results that distyryl aromatic systems may be particularly prone to undergo twofold dimerization reactions, and we can anticipate that such reactions will find application in the construction of a variety of novel, multi-cyclic systems.

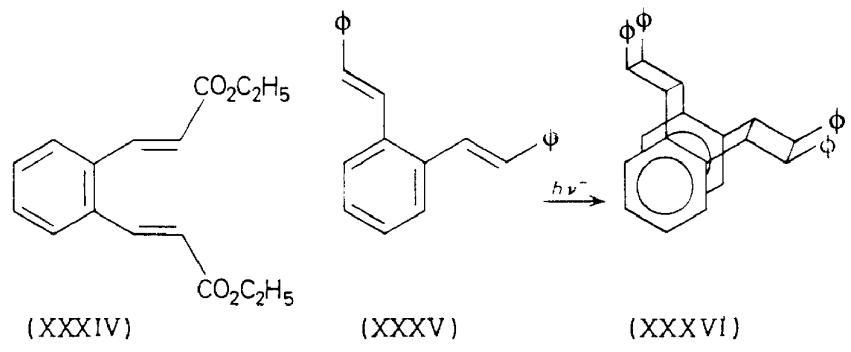

Of course, the transformation of (XXV) into (XXVIIa) tells nothing about the intramolecular cycloadditions of this substrate. In an effort to obtain monomeric products which could be compared with those derived from 1,8-divinylnaphthalene itself, (XXV) was irradiated in dilute solution. Under these conditions, a mixture of three crystalline photoisomers comprising (XXXVII) (40 per cent), (XXXVIII) (38 per cent) and (XXXIX) ( 5 per cent) could be isolated. These structural assignments were based chiefly on mass spectral and n.m.r. data. Thus, the two major products 
showed base peaks at $m / e$ 241, corresponding to $\left(\mathrm{M}-\mathrm{C}_{7} \mathrm{H}_{7}\right)$ fragments, best formulated as (XL) in analogy to the $\mathrm{M}-\mathrm{CH}_{3}$ ion characteristic of (XXIII). The minor component showed its base peak at $m / e$ 180, corresponding to loss of acenaphthalene from (XXXIX). The stereochemistry of each of these compounds can be deduced largely from n.m.r. spectral analysis, and

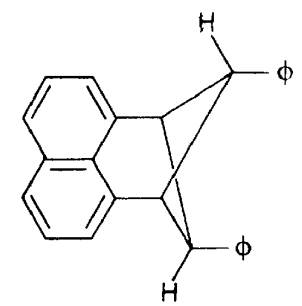

(XXXVII)

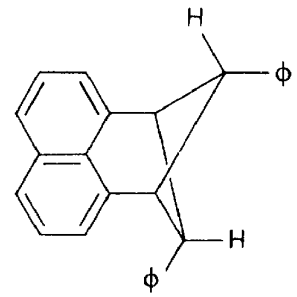

$(X X X V I I I)$

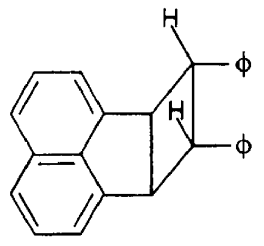

$(\mathrm{XXXIX)}$<smiles></smiles>

(XL)

was confirmed chemically by exhaustive ozonolysis. Thus, (XXXVII) gave trans,trans,trans-1,2,3,4-tetracarbomethoxycyclobutane, while (XXXVIII) and (XXXIX) gave the cis,trans,trans- and cis,trans,cis-isomers of this tetraester respectively.

The preference for head-to-tail cyclization in the case of (XXV) thus parallels the results obtained with divinylnaphthalene (XXII) itself. This preference finds a simple rationalization on the basis of the most favourable conformations for these 1,8-disubstituted naphthalenes. Thus, we would
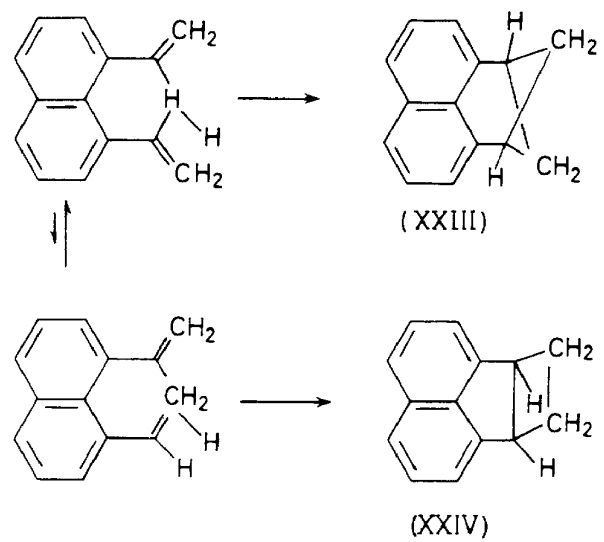
expect (XXII) to exist primarily with its vinyl groups projecting on opposite sides of the naphthalene ring, and the cyclization of this form to give (XXIII) involves a minimum amount of nuclear motion. Closure of the less important conformation, in which the two vinyl groups extend on the same side of the naphthalene ring system, should give the minor photoproduct.

The observation that this pattern is unchanged by the addition of terminal phenyl groups is striking, and suggests that the photocyclizations of (XXV) are also controlled largely by the same steric factors. If this constancy of reaction course proves general for other appended groupings, similar cyclizations should permit facile syntheses of 6-substituted peri-naphthobicyclo[3.1.1] heptenes of the general formulae (XLI) and (XLII), which offer interesting prospects for studies of carbonium ion reactions.

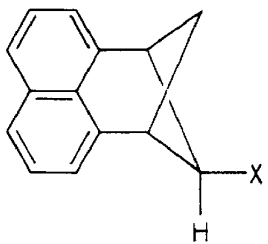

$(X L I)$

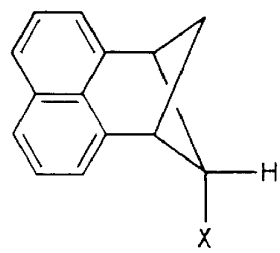

(XLII)

\section{ACKNOWLEDGEMENTS}

We welcome this opportunity to acknowledge our indebtedness to the National Science Foundation, the National Institutes of Health, and Hoffmann LaRoche, Inc., for their generous support of these studies.

\section{REFERENCES}

1 J. Meinwald, Record Chem. Prog. (Kresge-Hooker Sci. Lib.), 22, 39 (1961).

2 J. Meinwald and Y. C. Meinwald in Advances in Alicyclic Chemistry, Vol. I, pp 1-51, Academic Press : New York (1966).

3 J. Meinwald and F. Uno, J. Am. Chem. Soc. 90, 800 (1968).

4 R. Srinivasan, J. Phys. Chem. 67, 1367 (1963).

5 R. Srinivasan, J, Am. Chem. Soc. 83, 2806 (1961).

6 R. J. de Kock, N. G. Minnaard and E. Havinga, Rec. Trav. Chim. Pays-Bas, 79, 922 (1960).

7 K. Crowley, J. Am. Chem. Soc. 86, 5692(1964).

8 J. Meinwald, A. Eckell and K. Erickson, J. Am. Chem. Soc. 87, 3532 (1965).

9 J. Meinwald and P. H. Mazzocchi, J. Am. Chem. Soc. 88, 2850 (1966).

10 M. Pomerantz, J. Am. Chem. Soc. 89,695(1967).

11 J. Meinwald and P. H. Mazzocchi, J. Am. Chem. Soc. 89, 696 (1967).

12 J. K. Stille and R. T. Foster, J. Org. Chem. 28, 2703 (1963).

13 R. H. Mitchell and F. Sondheimer, Tetrahedron, 24, 1397 (1968).

14 Unpublished results with Dr M. Ikeda.

15 R. P. Houlton and W. Kemp, Tetrahedron Letters, 1045 (1968).

16 R. H. Mitchell and F. Sondheimer, J. Am. Chem. Soc. 90, 530 (1968).

17 E. Clar, K. F. Lang and H. Schulz-Kiesow, Chem. Ber. 88, 1520 (1955).

18 D. F. Tavares and W. H. Ploder, Tetrahedron Letters, 1567 (1970).

19 E. Müller, H. Meier and M. Sauerbier, Chem. Ber. 103, 1356 (1970). 